International Journal of Biomedicine | June 2021 - Volume 11, Issue Suppl_1: Abstracts from the Third Russian International Conference "Cryo-electron microscopy 2021: achievements and prospects"

\author{
POSTER ABSTRACT PRESENTATIONS
}

SESSION TITLE: ADVANCES IN EM TECHNOLOGY AND PROCESSING

DOI: 10.21103/JJBM.11.Suppl_1.P9

\title{
Abstract P-9: Excess of Nhp6 over Spt16/Pob3 is Necessary for Efficient Nucleosome Unfolding by yFACT
}

\author{
$\underline{\text { Anastasiia L. Sivkina }}^{1}$, Daria K. Malinina ${ }^{1}$, Alexey V. Feofanov ${ }^{1,2}$, \\ Vasily M. Studitsky ${ }^{1,3}$ \\ ${ }^{1}$ Biology Faculty, Lomonosov Moscow State University, Moscow, Russia \\ ${ }^{2}$ Institute of Bioorganic Chemistry, Moscow, Russia \\ ${ }^{3}$ Fox Chase Cancer Center, Philadelphia, Pennsylvania, USA
}

Background: DNA accessibility in chromatin is important for proper gene expression and is regulated by multiple factors. One of them is histone chaperone FACT, which conducts large-scale ATP-independent nucleosome unfolding that increases the accessibility of nucleosomal DNA. FACT binding results in dramatic DNA uncoiling from nucleosome, occurs without apparent loss of histones, and proceeds via an 'all-or-none' mechanism, but the detailed mechanism of this process is still unknown. FACT-dependent nucleosome unfolding modulates the accessibility of nucleosomal DNA, and it is an important function of FACT in the processes of transcription, DNA replication, and repair in vivo.

Methods: Nucleosome-positioning DNA sequences containing fluorescent labels (Cy3 and Cy5) at positions 13/91 or 35/112 from the nucleosome boundary were used. Nucleosomes were assembled by transfer of histone octamers from chicken chromatin to nucleosomal DNA during dialysis from $2 \mathrm{M}$ $\mathrm{NaCl}$. After dialysis nucleosomes were gel purified and used at a final concentration of $0.5 \mathrm{nM}$ for spFRET measurements or at $10 \mathrm{nM}$ for EMSA analysis. For complex formation nucleosomes were incubated in the presence of Spt16/Pob3 $(0.13 \mu \mathrm{M})$ and $\operatorname{Nhp} 6(1.3 \mu \mathrm{M})$ for $10 \mathrm{~min}$ at $30^{\circ} \mathrm{C}$ in buffer containing $17 \mathrm{mM}$ HEPES, 2mM Tris-HCl, 0.8mM Na3EDTA, 0.11 mM 2-mercaptoethanol, $150 \mathrm{mM} \mathrm{KCl}, 11 \mathrm{mM} \mathrm{NaCl}, 1.1 \%$ glycerin, $12 \%$ sucrose.

In spFRET microscopy analysis, the proximity ratio EPR from each nucleosome was calculated based on the intensity of the signals, corrected to the background and plotted as relative frequency distribution. Each plot was fitted as a sum of two Gaussians to describe two conformational states of nucleosomes. The 
fractions of nucleosomes in different states were estimated by calculating the surface of areas under the corresponding Gaussian peaks as a fraction of the total area of the plot.

Results: Here we report the results of our analysis of nucleosome unfolding by yeast FACT (yFACT) at different ratios of Spt16/Pob3 and Nhp6 using singleparticle Förster resonance energy transfer (spFRET) microscopy. Our analysis suggests that the optimal ratio of Spt16/Pob3 to Nhp6 for the most efficient FACT-dependent nucleosome unfolding is 1:10. Importantly, a mere increase in the concentration of FACT results in a decrease of the functional activity, suggesting that the formation of a functional complex having a certain stoichiometry of Spt16/Pob3 to Nhp6 is essential for efficient FACT-dependent nucleosome unfolding.

Conclusion: We determined that a certain ratio of Spt16/Pob3 to Nhp6 is essential for efficient FACT-dependent nucleosome unfolding, suggesting the formation of a functional FACT: Nhp6 complex.

Key Words: chromatin $・$ FACT $\bullet$ nucleosome $・$ remodeler

This work was supported by the Russian Science Foundation (Grant No. 1944-02013)

*Corresponding author: Anastasiia Sivkina.E-mail: anastasiia.sivkina@gmail.ru

International Journal of Biomedicine. 2021;11 Suppl 1: S14-15

doi: 10.21103/IJBM.11.Suppl_1.P9

(C)2021 International Medical Research and Development Corporation 